\title{
Priority-Based Access Schemes and Throughput Performance in Wireless Networks Exploiting Multibeam Antennas
}

\author{
Xin Li, Member, IEEE, Yimin Zhang, Senior Member, IEEE, and Moeness G. Amin, Fellow, IEEE
}

\begin{abstract}
Multibeam antennas (MBAs) are capable of supporting concurrent communications with multiple neighboring users in wireless networks and, thus, can improve the throughput over omnidirectional antennas and switched-beam directional antennas. The throughput performance, however, may degrade in the absence of a proper access scheme for user scheduling, and thus, collisions occur. The probability of having such collisions further increases in a multipath propagation environment, which is commonly encountered in a wireless network. Furthermore, there is an increased demand to deliver various types of data with differentiated quality and latency requirements. Thus, it is desirable to incorporate user/packet priority in the medium access control (MAC) protocol. In this paper, priority-based access schemes are proposed for wireless networks exploiting MBAs, respectively, in single-path and multipath environments. An analytical framework is then developed to analyze the node throughput performance when the proposed access scheme is used. The analytical and simulated results clearly show that, in contrast to a random-access scheme, where significant throughput degradation occurs in a multipath propagation environment, when the proposed access scheme is applied, the node throughput becomes a nondecreasing function of the offered load, and the degradation due to multipath is significantly mitigated.
\end{abstract}

Index Terms-Multibeam antennas (MBAs), multipath, priority, throughput, wireless medium access.

\section{INTRODUCTION}

$\mathbf{T}$ HE BROAD deployment of wireless networks and the fast growth of diverse services require performance enhancement, particularly the quality of service and the throughput. Conventional wireless networks use omnidirectional antennas, which reserve the spectrum over a large area and waste network resources. The use of directional antennas can mitigate this problem by improving spatial reuse and extending coverage [1]-[7]. A more advanced structure is the use of multibeam antennas (MBAs), which support concurrent communications with multiple neighboring users [2], [8]-[13].

Although the use of MBAs in wireless networks, by enabling concurrent communications, may significantly enhance the throughput over omnidirectional antennas or switched-beam directional antennas, the throughput performance degrades in multipath environments due to the increased probability of

Manuscript received October 28, 2008; revised February 11, 2009. First published March 16, 2009; current version published August 14, 2009. This work was supported in part by the National Science Foundation under Grant EEC-0332490. The review of this paper was coordinated by Dr. P. Lin.

The authors are with the Center for Advanced Communications, Villanova University, Villanova, PA 19085 USA (e-mail: xin.1@ villanova.edu; yimin.zhang@villanova.edu; moeness.amin@villanova.edu).

Digital Object Identifier 10.1109/TVT.2009.2017897 collision between signals transmitted from different neighboring users [13]-[16]. Multipath propagation is a commonly encountered phenomenon in most wireless systems. Specifically, multipath is rich (with a large number of paths and a high angular spread) in a typical wireless network where the nodes are located in indoor or low-altitude outdoor environments [1], [2], [14]. Therefore, the impact of multipath propagation should be taken into account in designing an appropriate access scheme as a part of the medium-access-control (MAC) protocol for an MBA system.

Conventional MAC protocols, such as those used in the IEEE 802.11 standard [17] and the amendment [18], are designed to exploit omnidirectional antennas and, thus, are not suitable when directional antennas or MBAs are used. Recently, a variety of directional antenna-based MAC protocols have been proposed (see [19]-[29]), along with a few for MBA-based MAC protocols [8], [11]. Most of them are modified from the IEEE 802.11 standard to support the use of directional antennas or MBAs. A few of them have considered the impact of multipath propagation.

Supporting the growing need for applications that demand real-time delivery with overall latency constraints becomes an important and challenging issue for wireless networks [30]-[36]. To provide differentiated services to packet transmissions with different latency requirements, a MAC protocol must adopt a certain mechanism to provide appropriate prioritybased medium access such that packets with higher priority can be transmitted in preference to those with lower priority [33]-[36]. When MBAs are employed in such an application scenario, therefore, it becomes necessary to appropriately incorporate priority-based access schemes into directional MAC (DMAC) protocols.

In this paper, we develop priority-based access schemes for wireless networks using MBAs [2]. The priority classes of attempted transmission neighboring nodes (ATNs) and the impact of multipath propagation are considered. To exploit the spatial dimensionality, which has significantly attracted less attention compared with temporal access, this paper focusses on medium access in the spatial domain. It is mentioned, however, that the joint use of spatial and temporal dimensions can be incorporated to expand the dimensionality for more flexible access. Furthermore, we develop an analytical framework that enables us to analyze the throughput performance for a network using MBAs operating in single-path and multipath propagation environments [2]. In our analysis, throughput performance is explicitly analyzed after the probability that the target node 
(TN) accepts an arbitrary number of ATNs is derived. By contrast, previous work on the throughput/capacity analysis of networks focused on those exploiting omnidirectional antennas [38]-[40], directional antennas (e.g., [41]), and MBAs (e.g., [8], [9], and [11]) in single-path propagation environments and adaptive antenna-based networks in multipath environments [15]. However, there is a lack of analytical performance evaluation of networks exploiting MBAs, particularly when they are operated in a multipath environment.

The rest of this paper is organized as follows: In Section II, we discuss the relevant work on MAC protocols using directional antennas or MBAs and consider some existing prioritybased access mechanisms. The system model is provided in Section III, where the antenna model and network assumptions, as well as throughput metric, are described. Section IV presents our wireless access schemes. The analytical throughput performance analysis is performed in Section V. Numerical and simulated results are presented in Section VI. Finally, we give our concluding remarks in Section VII.

\section{RELATED WORK}

\section{A. DMAC Protocols}

Thus far, a variety of directional antenna-based MAC protocols have been proposed (e.g., [19]-[29]). These protocols can generally be classified into two main categories: 1) randomaccess protocols and 2) scheduling protocols. The former can further be classified into three kinds: 1) Pure requestto-send/clear-to-send (RTS/CTS) protocols. For example, the DMAC protocol [19], which is based on IEEE 802.11 MAC, accommodates directional antennas by blocking the directions from which RTS/CTS packets are received and allowing transmission in the unblocked directions. For the MAC protocol proposed in [20], the handshake is built up with omnidirectional RTS/CTS packets. The location is then estimated by finding the sector with the strongest signal power so that the data packets can directionally be transmitted. The idle nodes listen in all directions. In the directional virtual carrier sensing (DVCS) protocol [21], each node caches the estimated angle of arrivals (AoAs) of all neighboring nodes when hearing any signal. The RTS is directionally transmitted if the location information of a TN is available. The directional network allocation vector (DNAV) is used to indicate the reserved directions by neighboring nodes. The multihop MAC [22] is based on the DMAC and DVCS protocols. It exploits the extended transmission range of directional antennas and establishes the directional-omnidirectional or directional-directional wireless links according to the channel status. In the circular-DMAC protocol [23], a circular directional RTS is consecutively transmitted at each switched beam, and each node maintains a location table to record its neighboring nodes such that the DNAV mechanism can be used to solve the hidden terminal and deafness problems. 2) Tone-based DMAC protocols. For instance, in the dual busy tone multiple access with directional antennas protocol [24], the transmission and reception busy tones are provided by an additional transceiver, and the RTS/CTS and DATA packets are directionally transmitted. A node defers to transmit/receive when a busy tone is sensed. The Tone-DMAC
[25] uses a time slot to transmit a tone signal and, thus, simplifies the system as transmission is needed at only a single frequency. For AoA-MAC [26], [27], the time is slotted, and each slot contains three minislots, i.e., the tone transmission period (each transmitter sends a tone to its intended receiver, and then, the receiver points its beam toward the sender with help of the AoA algorithm), packet transmission period, and acknowledgement (ACK) transmission period. If the transmitter does not receive the ACK, then it retransmits later. 3) Other DMAC protocols; for example, the explicit synchronization via intelligent feedback protocol [11] uses the embedded feedback to synchronize neighboring nodes and, thus, allows simultaneous transmission in different beams. To mitigate deafness, a control packet is transmitted in the remaining beams.

Another category of directional antenna-based MAC protocols is based on scheduling. For instance, the receiver-oriented multiple access protocol [28] can simultaneously form multiple beams for transmission or reception. In each time slot, the active nodes are equally divided into transmitters and receivers, and they couple together in pairs to maximize the throughput. Either end of the transmission can use the directional mode. In the directional transmission and reception algorithm protocol proposed in [29], time is divided into frames, and each frame is split into three subframes: 1) the neighbor discovery and handshaking period; 2) the neighbor discovery confirmation and data reservation period; and 3) the data transmission period. The location information is exchanged via directional scanning for neighboring nodes.

\section{B. Existing Priority-Based Access Mechanisms}

To provide prioritized services to packets that require realtime delivery, the MAC protocols need to incorporate some differentiated priority-based access mechanisms [30]-[36]. Such existing mechanisms fall into two general categories: 1) reservation-based schemes and 2) contention-based schemes. In reservation-based schemes, contention only occurs at the resource reservation phase. For example, in the multihop access collision avoidance with piggyback reservation protocol [30], a real-time connection is set up using a fast reservation approach. The first data packet of high priority makes reservations along the route to the destination. The packets and the ACKs carry real-time access information in the headers. Each node maintains a reservation table to keep track of the reserved window. Hence, collisions do not occur once the reservation is achieved. On the contrary, in the contention-based schemes, the packet access decision is locally made, and contention is probabilistically resolved. For instance, the black burst is used to help flows with high priority to contend for the channel [31]. When the channel is idle for a short period of time, the node with the higher priority sends a longer black burst to jam the channel, and thus, it is easier to fetch the channel. Those packets with a lower priority drop out of black-burst contention once they hear the black burst.

There are some other schemes that are based on the modifications of the IEEE 802.11 distributed coordination function to support prioritized services. Many of them focus on the adjustment of the backoff period in anticipation of priority classes 
distinguished by different contention window-generation functions [32]. In [33], the packet priority information is piggybacked in four-way handshake frames. By monitoring the transmitted packets, each node maintains an access table that is used to assess its relative priority class. The backoff interval is generated based on the access table. In [34], a priority access scheme using two narrowband busy tone signals was developed to ensure the access of packets with high priority. A distributed priority packet access protocol was proposed in [35], which divides the packet transmission into two phases: 1) control and 2) data. The separate control phase is allocated for the transmission of priority information, which guarantees prioritized packet access according to the priority classes.

\section{Remarks}

Despite the importance of considering prioritized packet transmission in a multipath propagation environment, little work has been done for directional antennas and MBAs in such scenarios. While some papers may have considered one of the two issues, to our best knowledge, none of the MAC protocols, including those previously mentioned, has been developed for directional antennas or MBAs to consider both issues. In addition, most of the previous work focuses on the temporal dimension, whereas the utilization of spatial dimensionality in the design of access schemes remains unmature. Our contributions in this paper lie in the development of priority-based access algorithms for a network exploiting MBAs in single-path and multipath environments and the performance evaluation in an analytical framework.

\section{SYSTEM MODEL}

\section{A. Antenna Model}

This paper is emphasized to exploit the spatial dimensionality offered by MBAs and to examine how such capability is affected by access schemes, as well as multipath propagation. For this purpose, we consider a scenario where a TN is equipped with an $M$-beam antenna array and each predefined beam spans an angle of $2 \pi / M$ rad. As such, the antenna can form $M$ nonoverlapping conical beams that collectively cover the entire azimuth plane. With conical beams, the antenna gain within the beam angular region keeps a constant value and drops to zero outside the beam region. Hence, the interferers outside a beam are filtered out and do not affect the transmission of the beam. Different beams can concurrently transmit to or receive from multiple nodes. However, no beams are allowed to transmit a signal while the other beams are receiving signals. Such an antenna model is commonly used and provides a convenient framework to analytically examine the node throughput performance [1], [2]. The consideration of more complex and expensive steerable antennas [13], [28], [29], which adaptively support tracking and interference nulling, is out of the scope of this paper.

\section{B. Network Assumptions}

This paper concentrates on both the development of a priority-based access scheme for an MBA in a multipath propagation environment and the analytical evaluation of its performance. To clearly demonstrate the offerings of MBAs and the proposed access scheme, we focus on the spatial dimension and assume that all nodes in a region of interest share a wireless channel (i.e., sharing the same frequency, code, or time channel). Moreover, the time is assumed to be slotted, and all data packets have the same length $T$. Data packets, including the newly generated and retransmitted packets, arrive at the $i$ th node according to a Poisson process with an arrival rate of $\lambda_{i}$ packets per second, and hence, the corresponding offered load of the node is $R_{i}=\lambda_{i} T$. Each arriving packet is intended for a single neighboring user, and each node has an infinite buffer. To illustrate the performance of access schemes, we focus on one-hop consideration. It is assumed that, for a TN, $N$ neighboring nodes, which may be source nodes or relay nodes, are independently and randomly located within the communication range of the TN with a uniform angular distribution.

Regardless of the propagation environment, when multiple signal arrivals originating from different ATNs fall into the same beam, collisions occur in the beam of a TN. As a result, this beam cannot successfully receive packets and, thus, does not contribute to the throughput of the TN. Only those beams that successfully receive packets contribute to node throughput. It is clear that, without a proper access scheme at the MAC layer, collisions can occur since each ATN randomly originates packet transmission. Furthermore, in a multipath environment, the signals transmitted from an ATN may fall into multiple beams of the TN and, thus, result in more frequent collisions. Therefore, using a MAC protocol with proper access scheme to resolve contention and avoid collision in data packets is very important to improve the node throughput.

It is assumed that, during a time slot, an ATN first sends an RTS, which may contain priority information, and, upon receiving a CTS from its $\mathrm{TN}$, transmits the data packet(s). If the CTS and/or ACK are not correctly received, then this node retries to send the RTS in the next time slot with a certain probability, provided that the channel is available at that time. According to the access algorithms proposed in Section IV, a TN that receives RTS packets first detects the ATNs to obtain individual occupancy information with proper contention resolution algorithms, e.g., [43] and [44]. The TN then determines the acceptance of the ATNs and directionally replies the corresponding CTS packets to the accepted packets. To achieve these functions, DMAC protocols (e.g., [19]-[36]) can be revisited to incorporate the aforementioned access framework and the priority information of each ATN. Due to space limitation, we focus on the access schemes, and hence, detailed descriptions of MAC and routing schemes are omitted. See [19]-[36] for further information.

\section{Throughput Metric}

For convenience, we use node throughput gain (NTG) [1], [2], [13], [16], [37] as the throughput metric, which is defined as the mean number of neighboring users accepted by a TN. In other words, NTG is the mean number of successful data links. This definition highlights the effects of access schemes and important MAC layer parameters and diminishes the physical 
layer characteristics. As such, it reflects the advantage of the MBAs over omnidirectional and single-beam directional antennas. For specific applications, the NTG can be mapped to real data rates. Therefore, the term of the NTG is closely relevant to the conventional definition of throughput, and both indicate the channel utilization of the employed access scheme.

\section{Medium Access Schemes}

Following the system model described in Section III, we propose two access schemes that are used for single-path and multipath environments, respectively. The priority information of packets transmitted from different ATNs is considered in both access schemes. Note that the emphasis in this paper is placed on the exploitation of the spatial dimension, which is complementary to the temporal dimension as considered in most existing work. Therefore, while it is beyond the scope of this paper, the access over joint spatial and temporal dimensions is expected to provide more flexibility and better performance.

\section{A. Single-Path Propagation Environment}

In a single-path environment, an RTS packet transmitted from an ATN arrives at its TN and falls into only one beam of the TN. To consider priority-based access at the TN, all the $n(\leq N)$ detected ATNs are nonascendingly sorted based on their priority classes to form a queue: $U_{1}, U_{2}, \ldots, U_{n}$, where $U_{1}$ has the highest priority, and $U_{n}$ has the lowest priority. Assume that the $n$ ATNs fall into $b$ beams, where $b \leq \min (n, M)$. In this case, the maximum number of ATNs that can be accepted by the $\mathrm{TN}$ is $b$. One possible access scheme for the TN to achieve the maximum NTG is given here.

1) Unconditionally accept $U_{1}$.

2) Accept $U_{2}$ only if it does not fall into the same beam occupied by $U_{1}$; otherwise, deny it.

3) For $i>2$, accept $U_{i}$ only if it does not fall into any beams occupied by the previously accepted ATNs; otherwise, deny it.

4) Update $i$ as $i \leftarrow i+1$. Then, repeat list item 3 until either $i>n$ holds or all the $b$ beams are occupied.

5) Deny all the remaining ATNs if there are any.

6) Respectively reply the CTS to each accepted ATN.

Clearly, this access scheme protects the accepted links by denying the ATNs that fall into a beam already occupied by an accepted link. As such, only the ATN with the highest priority is guaranteed to be accepted, whereas the acceptance of other ATNs is not guaranteed. Furthermore, as $n$ increases, $b$ asymptotically approaches $M$, which yields an NTG of $M$.

\section{B. Multipath Propagation Environment}

A wireless network is typically located in a multipath-rich environment [14]. In this case, an RTS packet transmitted from an ATN may propagate through multiple paths and fall into multiple beams of its TN. Consequently, collisions are likely to occur more frequently if a proper access scheme is not applied.

Without loss of generality, it is assumed that an RTS packet transmitted from each ATN arrives at the TN through $K$ quasi- stationary paths. Note that some or all of the $K$ paths may occupy the same beam at the TN. Therefore, this model inherently includes the case where the nodes have different numbers of propagation paths. In this case, each of the $n \leq N$ ATNs may occupy at most $B=\min (K, M)$ beams. Denote $B_{a}$ as the number of all beams that the signal arrivals from the $n$ ATNs occupy. It is obvious that $B_{a} \leq \min (K n, M)$.

To consider the priority of packets transmitted from the ATNs, the TN, similar to what it does in the single-path situation, nonascendingly sorts the $n$ detected ATNs according to their priority classes to form a queue: $U_{1}, U_{2}, \ldots, U_{n}$. The access scheme, in this case, is implemented by the TN as given here.

1) Unconditionally accept $U_{1}$.

2) Accept $U_{2}$ only if none of its paths falls into the beams occupied by $U_{1}$; otherwise, deny it.

3) For $i>2$, accept $U_{i}$ only if none of its paths falls into the $B_{\mathrm{oc}}$ beams, where $B_{\mathrm{oc}}$ is the number of beams occupied by the previously accepted ATNs. Update $B_{\text {oc }}$ to include the beams occupied by the newly accepted user.

4) Update $i$ as $i \leftarrow i+1$. Then, repeat list item 3 until either $B_{\mathrm{oc}}=B_{a}$ or $i>n$ holds.

5) Deny all the remaining ATNs if there are any.

6) Reply the respective CTS to each accepted ATN.

It can be seen that this access scheme protects the previously accepted links to a maximum extent in the sense that all the beams of an accepted ATN are unaffected by the newly accepted ATNs. That is, all the accepted ATNs individually have mutually exclusive occupancies. This keeps the access relatively simple, and the accepted ATNs may achieve beam diversity. Similar to the single-path case, only the user with the highest priority is guaranteed to be accepted.

\section{Throughrut ANALYSIS}

In this section, we analyze the throughput performance in an analytical framework when the priority-based access schemes, which were proposed in Section IV, are exploited. By deriving the probability that a TN accepts an arbitrary number of ATNs, we obtain the analytical NTG performance. Both single-path and multipath environments are considered.

\section{A. Single-Path Propagation Environment}

In a single-path environment, when the RTS packets of the $n$ ATNs fall into $b \leq \min (n, M)$ beams of the TN, the NTG is $b$ since each of the $b$ beams accepts one ATN. The probability that $n$ ATNs occupy $b$ beams of the TN can be expressed as

$$
P^{\mathrm{s}}(M, n, b)=\left(\begin{array}{c}
M \\
b
\end{array}\right) \frac{b ! \cdot \mathrm{S}(n, b)}{M^{n}}
$$

where $\left(\begin{array}{c}M \\ b\end{array}\right)$ denotes the combination operation, which represents the number of different ways of selecting $b$ out of $M$ available beams; $\mathrm{S}(n, b)=(1 / b !) \sum_{i=0}^{b-1}(-1)^{i}\left(\begin{array}{c}b \\ i\end{array}\right)(b-i)^{n}$ is the Stirling number of the second kind [42], which represents the number of different ways that $n$ ATNs fall into $b$ beams without empty beams; and the superscript "s" indicates the 
single-path case. Note that, for $b>n$, the foregoing probability is zero, because $\mathrm{S}(n, b)=0$. As a result, the mean number of accepted neighboring users in the presence of $n$ ATNs is

$$
G_{n}^{\mathrm{s}}(M)=\sum_{b=1}^{\min (M, n)} b \cdot P^{\mathrm{s}}(M, n, b) .
$$

For convenience, we define $p$ as the probability that each neighboring user sends an RTS packet at the beginning of a time slot ${ }^{1}$ and $P^{\mathrm{a}}(N, n, p)$ as the probability that $n$ ATNs of a TN simultaneously send RTS packets to the TN. Then, one can obtain

$$
P^{\mathrm{a}}(N, n, p)=\left(\begin{array}{c}
N \\
n
\end{array}\right) p^{n}(1-p)^{N-n}
$$

Additionally, when $p$ is sufficiently small, the time-domain MAC protocol is similar to the slotted ALOHA protocol, and the offered load $R$ can then be approximated by $N p$ [11]. As such, (3) can be written as

$$
P^{\mathrm{a}}(N, n, p)=\frac{R^{n} e^{-R}}{n !} .
$$

Consequently, the NTG in a single-path environment with the exploitation of the priority-based access is the expected number of accepted ATNs, which is expressed as

$$
G^{\mathrm{s}}(M, N, p)=\sum_{n=1}^{N} P^{\mathrm{a}}(N, n, p) \cdot G_{n}^{\mathrm{s}}(M)
$$

which shows that, when $n$ is sufficiently large (i.e., highoffered-load case), the NTG asymptotically approaches $M$ with probability 1 .

The advantage of using proper access is evident by comparing (5) with the counterpart in the absence of proper access. For example, the analytical NTG developed for the slotted ALOHA protocol, which is described in [37, eq. (5)], is significantly lower than (5). Numerical and simulated results of the performance comparison are provided in Section VI.

\section{B. Multipath Propagation Environment}

Based on the access scheme developed for multipath environments, which is described in Section IV, the throughput performance is analyzed hereinafter.

Denote $S$ as the number of accepted neighboring users by the TN. Clearly, $S \leq \min (n, M)$. Specifically, for $n \geq M$, the maximum throughput of $M$ may be achieved when there are at least $M$ ATNs, each of which falls into only one exclusive beam of the TN. For the convenience of discussion, we denote $Q=\left\{U_{S_{1}}, U_{S_{2}}, \ldots, U_{S_{S}}\right\}$ as a set consisting of the $S$ accepted neighboring users, where $S_{s}$ is the user index corresponding to

\footnotetext{
${ }^{1}$ The necessary condition for the origination of RTS packet(s) is the existence of a nonempty transmit buffer, which contains the newly generated packet(s) and/or packet(s) to be retransmitted. Therefore, the value of $p$ depends on both the traffic parameters, such as new packet arrival rate $\lambda_{i}$, and the MAC layer parameters, e.g., the probability of retransmission.
}

the $s$ th accepted user $1 \leq s \leq S$. From the proposed prioritybased access, the following constraints can be derived:

$$
\left\{\begin{array}{l}
S_{1}=1 \\
S_{s-1}+1 \leq S_{s} \leq n-(S-s), \quad 1<s \leq S .
\end{array}\right.
$$

Let $b_{s}$ be the number of beams occupied by the $s$ th accepted user. Then, the following relationship holds:

$$
S=\arg \max _{1 \leq S^{\prime} \leq \min (n, M)}\left\{\left(B_{a}-\sum_{s=1}^{S^{\prime}} b_{s}\right) \geq 0\right\}
$$

where $B_{a}$, as defined in Section IV-B, represents the total number of beams that the signal arrivals from the $n$ ATNs occupy. Denote $B_{s}$ as the maximum possible number of beams that an ATN can occupy, i.e., $1 \leq b_{s} \leq B_{s}, 1 \leq s \leq S$. Thus, when $S$ is obtained, $B_{s}$ is bounded by two factors: 1) the number of its paths and 2) the maximum number of allowed beams such that all the $S$ users do not mutually collide. As a result, we can express $B_{s}$ as

$$
\left\{\begin{array}{l}
B_{1}=\min (K, M-S+1) \\
B_{s}=\min \left(K, M-S+s-\sum_{i=1}^{s-1} b_{i}\right), \quad 1<s \leq S .
\end{array}\right.
$$

In the following, we consider the probability that $S$ out of $n$ ATNs are successfully accepted by the TN. We first derive the probability for the cases of $S=1$ and $S=2$, respectively, and the results are then generalized to the case of $S \geq 2$.

1) $S=1$ : For $S=1$, there is only one ATN being accepted by the TN. This event occurs when $b_{1} \geq 1$ beams occupied by $U_{1}$ are collision free, whereas at least one path of each of the remaining $n-1$ ATNs in the queue falls into one of the beams occupied by $U_{1}$. Therefore, the probability that only one ATN is accepted by the TN can be written as

$$
P^{\mathrm{m}}(M, n, 1, K)=\sum_{b_{1}=1}^{B_{1}} P\left(M, b_{1}, K\right) \cdot\left(\frac{M^{K}-\left(M-b_{1}\right)^{K}}{M^{K}}\right)^{n-1}
$$

where

$$
P(M, b, K)={ }^{M} P_{b} \frac{\mathrm{S}(K, b)}{M^{K}}
$$

is the probability that all the $K$ paths originating from one user fall into $b$ out of $M$ beams of the TN, ${ }^{M} P_{b}$ denotes the permutation operation representing the number of different ways to select $b$ beams from all the $M$ beams, and the Stirling number $\mathrm{S}(K, b)$ represents the number of different ways that $K$ paths fall into $b$ beams without empty beams. The subscript " $m$ " indicates the multipath case. Note that, mathematically, $P(M, b, K)$ in (10) is equal to $P^{\mathrm{s}}(M, K, b)$ given in (1).

It can be shown that, as $K \rightarrow \infty,((b-i) / M)^{K}$ approaches 0 , except for $b=M$ and $i=0$. As a result

$$
\begin{aligned}
P(M, M, K) & =\frac{M ! \cdot S(K, M)}{M^{K}} \\
& =\sum_{i=0}^{M-1}(-1)^{i}\left(\begin{array}{c}
M \\
i
\end{array}\right)\left(1-\frac{i}{M}\right)^{K} \stackrel{K \rightarrow \infty}{\longrightarrow} 1 .
\end{aligned}
$$


That is, as $K$ increases, each ATN is likely to occupy all the $M$ beams, which results in a unit node throughput.

2) $S=2:$ For convenience, let

$$
\begin{aligned}
P\left(M, b_{i}, b_{j}, K\right) & =P\left(M-b_{i}, b_{j}, K\right)\left(\frac{M-b_{i}}{M}\right)^{K} \\
& ={ }^{M-b_{i}} P_{b_{j}} \frac{\mathrm{S}\left(K, b_{j}\right)}{M^{K}}
\end{aligned}
$$

represent the probability that all $K$ paths of $U_{j}$ fall into $b_{j}$ out of $M-b_{i}$ beams of the TN. If only two ATNs are accepted by the TN, i.e., $S=2$, the second accepted user $\left(U_{S_{2}}\right)$ has a user index $i=S_{2}$ that may range over $[2, n]$. In this case, $B_{1}=\min (K, M-1)$, and $B_{2}=\min \left(K, M-b_{1}\right)$. We first consider a relatively simple case where, in addition to $U_{1}$, $U_{2}$ is also accepted, i.e., $S_{2}=2$. The number of beams that $U_{2}$ occupies is denoted $b_{2}$, where $1 \leq b_{2} \leq B_{2}$. Thus, when $U_{2}, U_{3}, \ldots, U_{n}$ fall into the remaining $M-b_{1}$ beams that are not occupied by $U_{1}$, the conditional acceptance probability of $U_{S_{2}}$ can be expressed as

$$
\begin{aligned}
P_{S_{2}}^{\mathrm{c}}\left(M, n, b_{1}, K\right)= & \sum_{b_{2}=1}^{B_{2}} P\left(M, b_{1}, b_{2}, K\right) \\
& \cdot\left(\frac{M^{K}-\left(M-b_{1}-b_{2}\right)^{K}}{M^{K}}\right)^{n-2} .
\end{aligned}
$$

Note that $P_{S_{2}}^{\mathrm{c}}(\cdot)$ is a function of $M, n, b_{1}, K$, and $S_{2}$. For clarity, we omit these variables thereafter. Next, we consider the case where $U_{2}, \ldots, U_{S_{2}-1}$ are denied and $U_{S_{2}}$ is accepted, where $2<S_{2} \leq n$. Similarly, $U_{S_{2}}$ may fall into $b_{2}$ beams, with $1 \leq b_{2} \leq B_{2}$. In this case, the conditional acceptance probability of $U_{S_{2}}$, where users $U_{S_{2}}, \ldots, U_{n}$ fall into the $M-b_{1}$ beams that are not occupied by user $U_{1}$, is given by

$$
P_{S_{2}}^{\mathrm{c}}=\sum_{b_{2}=1}^{B_{2}} P\left(M, b_{1}, b_{2}, K\right) \cdot\left(\frac{M^{K}-\left(M-b_{1}-b_{2}\right)^{K}}{M^{K}}\right)^{n-S_{2}} .
$$

It is evident that (13) is just the special case of (14) for $S_{2}=2$.

From (14) and by further considering the probability that $U_{2}, \ldots, U_{S_{2}-1}$ are denied, where $U_{2}, U_{3}, \ldots, U_{S_{2}}, \ldots, U_{n}$ fall into the remaining $M-b_{1}$ beams that are not occupied by $U_{1}$, one can obtain the final acceptance probability of $U_{S_{2}}$ as

$$
P_{S_{2}}^{\mathrm{m}}=\sum_{S_{2}=2}^{n} P_{S_{2}}^{\mathrm{c}} \cdot\left(\frac{M^{K}-\left(M-b_{1}\right)^{K}}{M^{K}}\right)^{S_{2}-S_{1}-1} .
$$

Consequently, the probability that only two ATNs are accepted by the TN can be written as

$$
P^{\mathrm{m}}(M, n, 2, K)=\sum_{b_{1}=1}^{B_{1}} P\left(M, b_{1}, K\right) \cdot P_{S_{2}}^{\mathrm{m}} .
$$

3) $S>2$ : Similarly, for $S>2$, the probability that $S$ ATNs are accepted by the TN can also be derived. From (14), one can find that, when $U_{S_{S}}, \ldots, U_{n}$ fall into the remaining $M-$
$\sum_{s=1}^{S-1} b_{s}$ beams that are not occupied by the $S-1$ previously accepted users, the conditional acceptance probability of $U_{S_{S}}$ can be expressed as

$$
\begin{aligned}
P_{S_{S}}^{\mathrm{c}}=\sum_{b_{S}=1}^{B_{S}} P\left(M, \sum_{s=1}^{S-1} b_{s}, b_{S}, K\right) \\
\cdot\left(\frac{M^{K}-\left(M-\sum_{s=1}^{S} b_{s}\right)^{K}}{M^{K}}\right)^{n-S_{S}} .
\end{aligned}
$$

Following (15), when $U_{S_{S-1}+1}, \ldots, U_{n}$ fall into the remaining $M-\sum_{s=1}^{S-1} b_{s}$ beams that are not occupied by the $S-1$ previously accepted users, the final acceptance probability of $U_{S_{S}}$ can be written as

$$
P_{S_{S}}^{\mathrm{m}}=\sum_{S_{S}=S_{S-1}+1}^{n} P_{S_{S}}^{\mathrm{c}} \cdot\left(\frac{M^{K}-\left(M-\sum_{s=1}^{S-1} b_{s}\right)^{K}}{M^{K}}\right)^{S_{S}-S_{S-1}-1}
$$

When $U_{S_{S-1}}, \ldots, U_{n}$ fall into the remaining $M-\sum_{s=1}^{S-2} b_{s}$ beams that are not occupied by the $S-2$ previously accepted users, similar to (17), one can obtain the conditional acceptance probability of $U_{S_{S-1}}$ as

$$
P_{S_{S-1}}^{\mathrm{c}}=\sum_{b_{S-1}=1}^{B_{S-1}} P\left(M, \sum_{s=1}^{S-2} b_{s}, b_{S-1}, K\right) \cdot P_{S_{S}}^{\mathrm{m}} .
$$

Similarly, from (18), when $U_{S_{S-2}+1}, \ldots, U_{n}$ fall into the remaining $M-\sum_{s=1}^{S-2} b_{s}$ beams, the final acceptance probability of $U_{S_{S-1}}$ can be written as

$$
\begin{aligned}
P_{S_{S-1}}^{\mathrm{m}}= & \sum_{S_{S-1}=S_{S-2}+1}^{n} P_{S_{S-1}}^{\mathrm{c}} \\
& \cdot\left(\frac{M^{K}-\left(M-\sum_{s=1}^{S-2} b_{s}\right)^{K}}{M^{K}}\right)^{S_{S-1}-S_{S-2}-1} .
\end{aligned}
$$

The derivation process can be continued in a similar way. Finally, one can find the conditional acceptance probability of $U_{S_{2}}$, where $U_{S_{2}}, \ldots, U_{n}$ fall into the $M-b_{1}$ beams that are not occupied by $U_{1}$, which is expressed by

$$
P_{S_{2}}^{\mathrm{c}}=\sum_{b_{2}=1}^{B_{2}} P\left(M, b_{1}, b_{2}, K\right) \cdot P_{S_{3}}^{\mathrm{m}} .
$$

Therefore, when $U_{2}, \ldots, U_{n}$ fall into the remaining $M-b_{1}$ beams, one can also obtain the final acceptance probability of $U_{S_{2}}$ as

$$
P_{S_{2}}^{\mathrm{m}}=\sum_{S_{2}=2}^{n} P_{S_{2}}^{\mathrm{c}} \cdot\left(\frac{M^{K}-\left(M-b_{1}\right)^{K}}{M^{K}}\right)^{S_{2}-S_{1}-1} .
$$

As a result, the probability that $U_{1}, \ldots, U_{n}$ fall into $M$ beams and only $S$ ATNs are successfully accepted by the TN can be 
expressed as

$$
P^{\mathrm{m}}(M, n, S, K)=P_{S_{1}}^{\mathrm{c}}=\sum_{b_{1}=1}^{B_{1}} P\left(M, b_{1}, K\right) \cdot P_{S_{2}}^{\mathrm{m}} .
$$

Once the probability is obtained from (23) for all the valid values of $S$, the mean number of accepted neighboring users by the TN in the presence of $n$ ATNs can be obtained as

$$
G_{n}^{\mathrm{m}}(M, K)=\sum_{S=1}^{\min (M, n)} S \cdot P^{\mathrm{m}}(M, n, S, K) .
$$

Consequently, the NTG in a multipath environment with the exploitation of the priority-based access scheme is given by

$$
G^{\mathrm{m}}(M, N, K, p)=\sum_{n=1}^{N} P^{\mathrm{a}}(N, n, p) \cdot G_{n}^{\mathrm{m}}(M, K)
$$

where $P^{\mathrm{a}}(N, n, p)$ is a function of the offered load, as given in (3) and (4).

In contrast, the analytical NTG in the absence of proper access schemes is provided in [16, eq. (24)], which is explicitly different from (25). Numerical and simulation comparisons of the NTG with and without the use of the proposed access scheme are presented in Section VI.

\section{Numerical and Simulated Results}

In this section, numerical and simulated results are provided to depict the accuracy of the theoretical results developed in the previous section and to display the significance of using the proposed access schemes. Two cases are considered, where the number of beams is four $(M=4)$ and eight $(M=8)$, respectively. The number of paths $K$ varies from 1 to 6 , and the offered load $R$ varies from 0 to 20 . The probability that each neighboring user attempts to originate an RTS packet is assumed to be $p=0.1$. Note that, as mentioned in Section V-A, the offered load depends on the product of $N p$ and is nearly independent of $p$ when $p$ is small. Each simulation result is obtained using 1000 consecutive time slots. For specific MAC layer parameters, the simulated NTG depicted in this section can accordingly be mapped to the available data rates.

\section{A. Throughput With Priority-Based Access Schemes}

When the proposed access schemes are applied, the analytical and simulated NTG results of a TN in a network in various multipath environments are plotted in Figs. 1 and 2, respectively, for four- and eight-beam antennas. Both figures show a good agreement between the simulated and analytical results. With the exploitation of the proposed access schemes, NTG becomes a nondecreasing function of the offered load, i.e., the throughput performance is not degenerated at oversaturated offered loads. However, the presence of multipath propagation reduces the throughput. Comparing Figs. 1 and 2, it is clear that the NTG improves as the number of beams increases, because a higher number of available beams can concurrently support

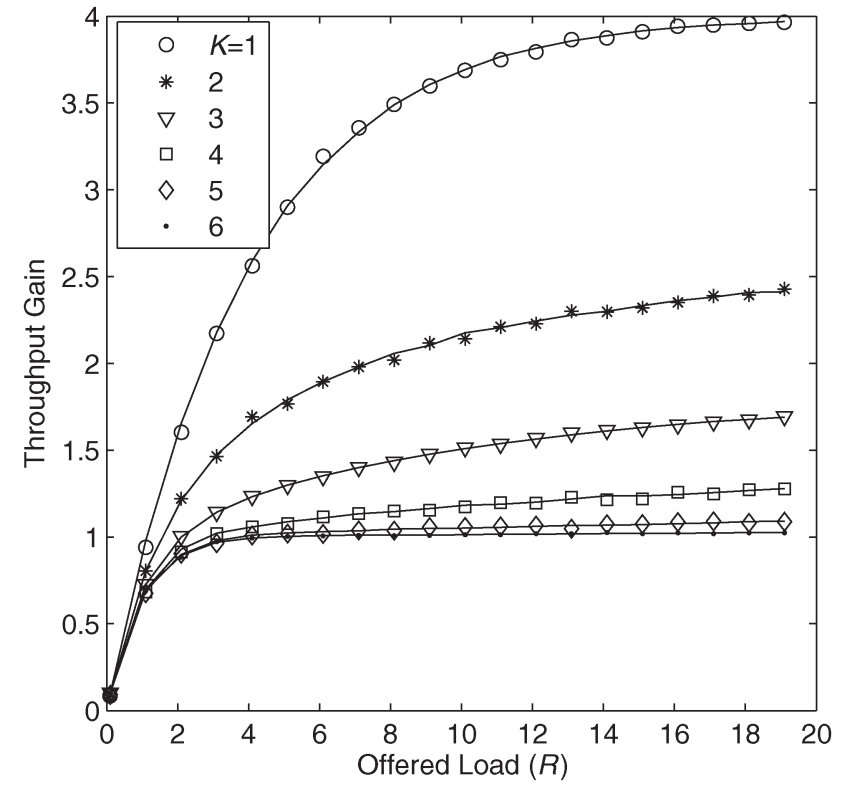

Fig. 1. (Markers) Analytical and (lines) simulated NTG of a TN exploiting a four-beam antenna with the use of the proposed access schemes in various multipath environments.

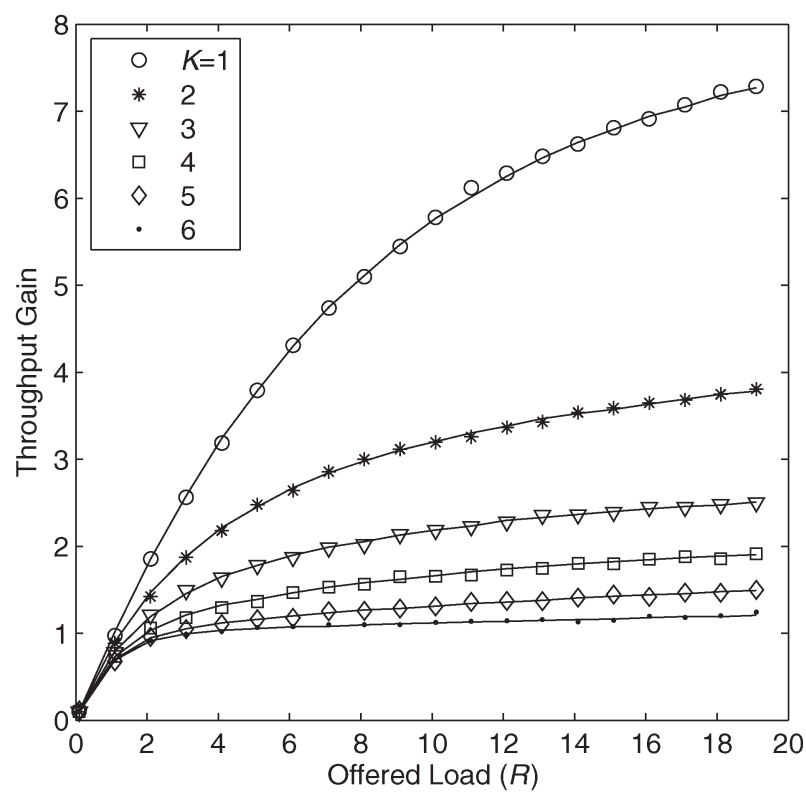

Fig. 2. (Markers) Analytical and (lines) simulated NTG of a TN exploiting an eight-beam antenna with the use of the proposed access schemes in various multipath environments.

more users. This can easily be expected and coincides with previous observations [38]. A rather more important fact is observed in Figs. 1 and 2, where the NTG in the case of $K=3$ and $M=8$ is comparable with that in the case of $K=2$ and $M=4$. That is, from the NTG perspective, the contribution of using a higher number of beams is nullified by the destructive impact of severe multipath.

Furthermore, the impact of the number of multipaths on the NTG is illustrated in Figs. 3 and 4 at different values of the offered load $R$. We consider the number of beams $M=4$ with typical values $R=4,8$, and 12 in Fig. 3 and $M=8$ with $R=4,8,12$, and 16 in Fig. 4, respectively. It is shown that, 


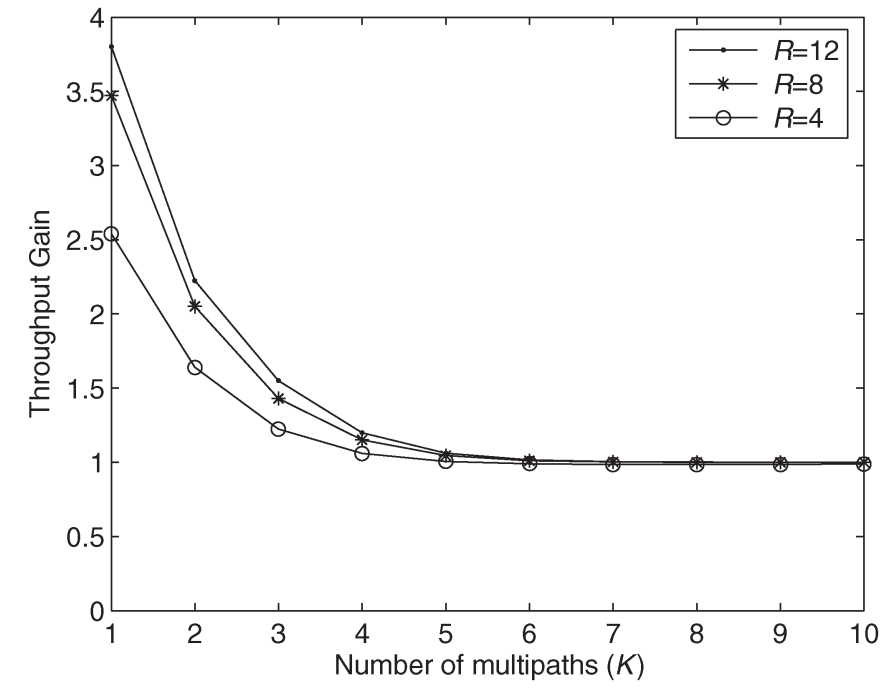

Fig. 3. Impact of the number of multipaths on the NTG of a TN exploiting a four-beam antenna with the use of the proposed access schemes.

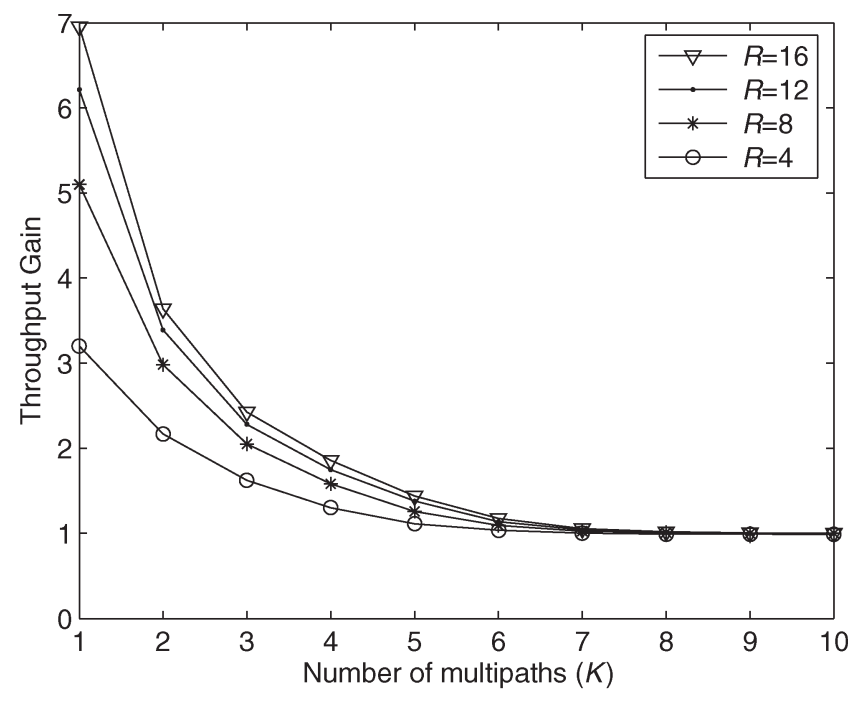

Fig. 4. Impact of the number of multipaths on the NTG of a TN exploiting an eight-beam antenna with the use of the proposed access schemes.

even with the use of the access schemes, the NTG remains a decreasing function with respect to the number of multipaths. That is, at a given value of the offered load, for a higher number of multipaths, more ATNs can be denied. In particular, the saturated NTG corresponding to a very large value of $K$ approaches unity, i.e., only one ATN with the highest priority is accepted. This result verifies the discussion we made in Section $\mathrm{V}$ that, as $K$ increases, each node is likely to occupy all the beams, and thus, a unit NTG is yielded. Moreover, both figures show that, at a moderate number of multipaths, the relative throughput degeneration is more severe at a higher value of the offered load.

\section{B. Throughput Comparison With/Without Proposed Schemes}

For comparison, the analytical NTG results of a TN with and without the use of the proposed access are plotted in Figs. 5 and 6 , respectively, for four- and eight-beam antennas in multipath environments. Both figures illustrate that, as opposed to

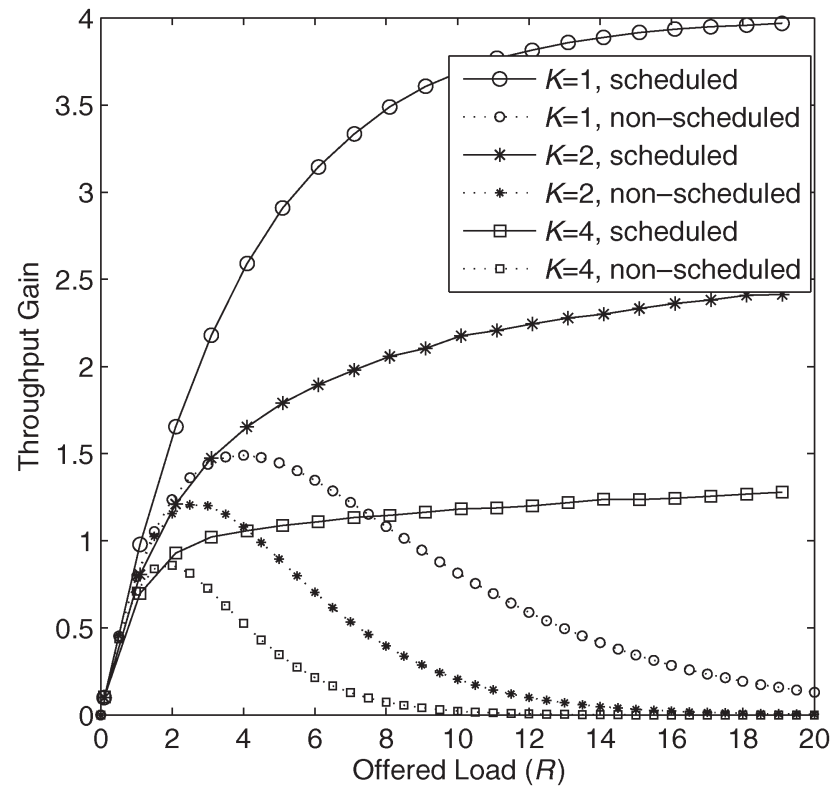

Fig. 5. Analytical NTG of a TN exploiting a four-beam antenna with and without the use of the proposed access schemes in multipath environments.

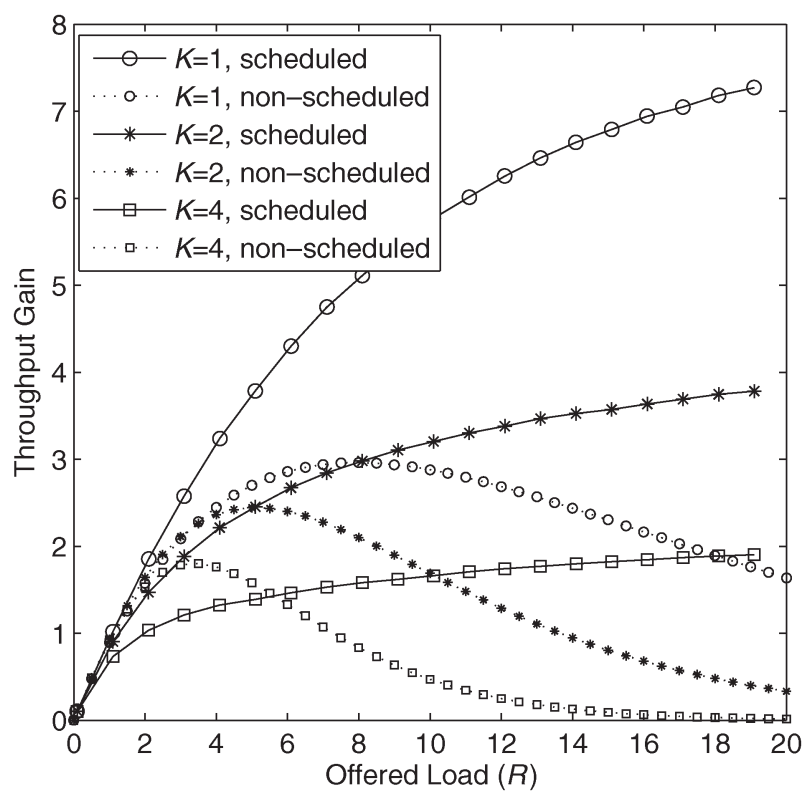

Fig. 6. Analytical NTG of a TN exploiting an eight-beam antenna with and without the use of the proposed access schemes in multipath environments.

scenarios where the proposed access schemes are used ("scheduled"), the NTG is not a monotonically increasing function of the offered load in the absence of the proposed access schemes ("nonscheduled") [16]. When the offered load is higher than a threshold value, the NTG decreases since a higher value of offered load increases the likelihood of collision. Moreover, the threshold value of the offered load decreases as the number of multipaths increases. This is due to the fact that, for a given offered load, more multipaths lead to more frequent collisions and, hence, a lower threshold value.

Furthermore, as depicted in Figs. 5 and 6, it is observed by comparing the respective pairs of NTG with and without the use of the proposed access that, in a single-path environment, 
the NTG with the proposed access is higher than that without access. However, in a multipath environment, it does not always hold true. Specifically, in the case of a relatively low offered load, the NTG for the case of "nonscheduled" is higher. This is due to the fact that the access schemes proposed in this paper have considered the priority classes of ATNs, which can penalize the NTG. A similar phenomenon in which the access penalizes the throughput at low offered load was also observed in [8], where the carrier-sense multiple-access protocol was employed without considering both the priority classes and the multipath impact.

\section{CONCLUSION}

In this paper, we have proposed two priority-based access schemes for networks exploiting MBAs, which correspond to single-path and multipath propagation environments, respectively. The proposed access schemes focus on the spatial domain and demonstrate the advantages of exploiting concurrent communications through multiple spatial-domain channels provided by the MBAs. In our model, time is assumed to be slotted, and the wireless channel has a single occupancy in terms of frequency or coding. An analytical framework for the throughput performance evaluation of a wireless network using the proposed access schemes was developed. The important parameters, including priority classes and the number of propagation paths, as well as the offered load, were incorporated in the analysis. The probability that a TN accepts an arbitrary number of ATNs was derived, and the throughput performance was then explicitly analyzed. Simulation results showed good agreement with the analytical performance. It was demonstrated that the existence of multipath propagation always degrades the throughput performance. Such degradation, however, can be relieved with the use of the proposed access scheme. With the use of the proposed access schemes, throughput becomes a nondecreasing function with respect to the offered load, regardless of the number of propagation paths. In contrast with the slotted ALOHA protocol, the proposed schemes consistently provide improved throughput performance, particularly in high-offeredload situations.

\section{REFERENCES}

[1] X. Li, Y. Zhang, and M. G. Amin, "Node throughput analysis of decentralized wireless networks using multibeam antennas in multipath environments," Wireless Pers. Commun. [Online]. Available: http://www. springerlink.com/content/320t237q837v188r/fulltext.pdf

[2] X. Li, Y. Zhang, and M. G. Amin“"Throughput analysis of ad hoc networks using multibeam antennas with priority-based channel access scheduling," in Proc. IEEE Wireless Commun. Netw. Conf., Mar. 2008, pp. 1651-1655.

[3] S. Bandyopadhyay, S. Roy, and T. Ueda, Enhancing the Performance of Ad Hoc Wireless Networks With Smart Antennas. Boca Raton, FL: Auerbach, 2006.

[4] Z. Huang and C. Shen, "A comparison study of omnidirectional and directional MAC protocols for ad hoc networks," in Proc. IEEE GLOBECOM, Nov. 2002, vol. 1, pp. 57-61.

[5] T. Elbatt, T. Anderson, and B. Ryu, "Performance evaluation of multiple access protocols for ad hoc networks using directional antennas," in Proc. IEEE Wireless Commun. Netw. Conf., Mar. 2003, vol. 2, pp. 982-987.

[6] S. Yi, Y. Pei, and S. Kalyanaraman, "On the capacity improvement of ad hoc wireless networks using directional antennas," in Proc. ACM MobiCom, Jun. 2003, pp. 108-116.
[7] R. Ramanathan, J. Redi, and C. Santivanez, "Ad hoc networking with directional antennas: A complete system solution," IEEE J. Sel. Areas Commun., vol. 23, no. 3, pp. 496-506, Mar. 2005.

[8] D. Lal, V. Jain, Q. Zeng, and D. P. Agrawal, "Performance evaluation of medium access control for multiple-beam antenna nodes in a wireless LAN," IEEE Trans. Parallel Distrib. Syst., vol. 15, no. 12, pp. 1117-1129, Dec. 2004.

[9] A. Singh, P. Ramanathan, and B. V. Veen, "Spatial reuse through adaptive interference cancellation in multi-antenna wireless networks," in Proc. IEEE GLOBECOM, Nov. 2005, vol. 5, pp. 3092-3096.

[10] Z. Huang and C. Shen, "Multibeam antenna-based topology control with directional power intensity for ad hoc networks," IEEE Trans. Mobile Comput., vol. 5, no. 5, pp. 508-517, May 2006.

[11] V. Jain and D. P. Agrawal, "Concurrent packet reception bounds for ondemand MAC protocols for multiple beam antennas," in Proc. IEEE PIMRC, Sep. 2006, pp. 1-5.

[12] S. M. Das, H. Pucha, D. Koutsonikolas, Y. C. Hu, and D. Peroulis, "DMesh: Incorporating practical directional antennas in multi-channel wireless mesh networks," IEEE J. Sel. Areas Commun., vol. 24, no. 11, pp. 2028-2039, Nov. 2006.

[13] Y. Zhang, X. Li, and M. G. Amin, "Multi-channel smart antennas in wireless networks," in Proc. Asilomar Conf. Signals, Syst., Comput., Pacific Grove, CA, Oct./Nov. 2006, pp. 305-309.

[14] J. H. Winters, "Smart antenna techniques and their application to wireless ad hoc networks," IEEE Wireless Commun., vol. 13, no. 4, pp. 77-83, Aug. 2006.

[15] F. Babich, M. Comisso, M. D’Orlando, and L. Mania, "Interference mitigation on WLANs using smart antennas," Wirel. Pers. Commun. J., vol. 36, no. 4, pp. 387-401, Mar. 2006.

[16] Y. Zhang, X. Li, and M. G. Amin, "Mobile ad hoc networks exploiting multi-beam antennas," in Handbook on Advancements in Smart Antenna Technologies for Wireless Networks, C. Sun, J. Cheng, and T. Ohira, Eds., Hersey, PA: IGI Global, 2008.

[17] IEEE Standard for Wireless LAN Medium Access Control (MAC) and Physical Layer (PHY) Specifications, IEEE Std. 802.11, 1997.

[18] IEEE Standard for Wireless LAN Medium Access Control (MAC) and Physical Layer (PHY) Specifications, Part 11, Medium Access Control (MAC) Quality of Service Enhancements, Amendment 8, IEEE Std. 802.11e, 2005

[19] Y. B. Ko, V. Shankarkumar, and N. H. Vaidya, "Medium access control protocols using directional antennas in ad hoc networks," in Proc. IEEE INFOCOM, Mar. 2000, vol. 1, pp. 13-21.

[20] A. Nasipuri, S. Ye, J. You, and R. E. Hiromoto, "A MAC protocol for mobile ad hoc networks using directional antennas," in Proc. IEEE Wireless Commun. Netw. Conf., Sep. 2000, vol. 3, pp. 1214-1219.

[21] M. Takai, J. Martin, A. Ren, and R. Bagrodia, "Directional virtual carrier sensing for directional antennas in mobile ad hoc networks," in Proc. ACM MobiHoc, Jun. 2002, pp. 183-193.

[22] R. R. Choudhury, X. Yang, R. Ramanathan, and N. H. Vaidya, "On designing MAC protocols for wireless networks using directional antennas," IEEE Trans. Mobile Comput., vol. 5, no. 5, pp. 477-490, May 2006.

[23] T. Korakis, G. Jakllari, and L. Tassiulas, "A MAC protocol for full exploitation of directional antennas in ad hoc wireless networks," in Proc. ACM MobiHoc, Jun. 2003, pp. 98-107.

[24] Z. Huang, C. Shen, C. Srisathapornphat, and C. Jaikaeo, "A busy-tone based directional MAC protocol for ad hoc networks," in Proc. IEEE MILCOM, Oct. 2002, vol. 2, pp. 1233-1238.

[25] R. R. Choudhury and N. H. Vaidya, "Deafness: A MAC problem in ad hoc networks when using directional antennas," in Proc. IEEE Int. Conf. Netw. Protocols, Oct. 2004, pp. 283-292.

[26] H. Singh and S. Singh, "A MAC protocol based on adaptive beamforming for ad hoc networks," in Proc. IEEE PIMRC, 2003, pp. 1346-1350.

[27] H. Singh and S. Singh, "Tone based MAC protocol for use with adaptive array antennas," in Proc. IEEE Wireless Commun. Netw. Conf., 2004, pp. 1246-1251

[28] L. Bao and J. Aceves, "Transmission scheduling in ad hoc networks with directional antennas," in Proc. ACM MobiCom, Jun. 2002, pp. 48-58.

[29] Z. Zhang, "Pure directional transmission and reception algorithms in wireless ad hoc networks with directional antennas," in Proc. IEEE ICC, Jun. 2005, vol. 5, pp. 3386-3390.

[30] C. R. Lin and M. Gerla, "Real-time support in multihop wireless networks," Wireless Networks, vol. 5, no. 2, pp. 125-135, Mar. 1999.

[31] J. L. Sobrinho and A. S. Krishnakumar, "Quality-of-service in ad hoc carrier sense multiple access networks," IEEE J. Sel. Areas Commun., vol. 17, no. 8, pp. 1353-1368, Aug. 1999. 
[32] A. Veres, A. T. Campbell, M. Barry, and L.-H. Sun, "Supporting service differentiation in wireless packet networks using distributed control," IEEE J. Sel. Areas Commun., vol. 19, no. 10, pp. 2081-2093, Oct. 2001.

[33] V. Kanodia, C. Li, A. Sabharwal, B. Sadeghi, and E. Knightly, "Distributed multi-hop scheduling and medium access with delay and throughput constraints," in Proc. ACM MobiCom, Jul. 2001, vol. 1, pp. 200-209.

[34] X. Yang and N. H. Vaidya, "Priority scheduling in wireless ad hoc networks," in Proc. ACM MobiHoc, Jun. 2002, pp. 71-79.

[35] J. Yin, Q.-A. Zeng, and D. P. Agrawal, "A novel priority based scheduling scheme for ad hoc networks," in Proc. IEEE VTC, Oct. 2003, vol. 3, pp. 1637-1641.

[36] Y. Xiao, "A simple and effective priority scheme for IEEE 802.11," IEEE Commun. Lett., vol. 7, no. 2, pp. 70-72, Feb. 2003.

[37] A. Chockalingam and R. R. Rao, "MAC layer performance with steerable multibeam antenna arrays," in Proc. IEEE PIMRC, Sep. 1998, vol. 2, pp. 973-977.

[38] P. Gupta and P. R. Kumar, "The capacity of wireless networks," IEEE Trans. Inf. Theory, vol. 46, no. 2, pp. 388-404, Mar. 2000.

[39] J. Li, C. Blake, D. S. J. Decouto, H. I. Lee, and R. Morris, "Capacity of ad hoc wireless networks," in Proc. ACM MobiCom, Jul. 2001, pp. 61-69.

[40] S. Toumpis and A. J. Goldsmith, "Capacity regions for wireless ad hoc networks," IEEE Trans. Wireless Commun., vol. 2, no. 4, pp. 736-748, Jul. 2003.

[41] A. Spyropoulos and C. S. Raghavendra, "Capacity bounds for ad-hoc networks using directional antennas," in Proc. ICC, May 2003, vol. 1, pp. 348-352.

[42] R. L. Graham, D. E. Knuth, and O. Patashnik, Concrete Mathematics: A Foundation for Computer Science, 2nd ed. Reading, MA: AddisonWesley, 1994.

[43] X. Li, "Contention resolution in random-access wireless networks based on orthogonal complementary codes," IEEE Trans. Commun., vol. 52, no. 1, pp. 82-89, Jan. 2004.

[44] J. Wang, Y. Fang, and D. Wu, "Enhancing the performance of medium access control for WLAN with multibeam access point," IEEE Trans. Wireless Commun., vol. 6, no. 2, pp. 556-565, Feb. 2007.

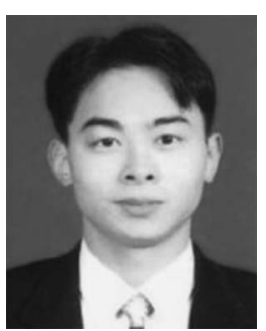

Xin Li (M'08) received the B.S. degree in electrical engineering and the M.S. degree in information and communications engineering from Chongqing University of Posts and Telecommunications, Chongqing, China, in 1999 and 2002, respectively, and the Ph.D. degree in information and communications engineering and electromagnetic theory from the University of Electronic Science and Technology of China, Chengdu, China, in 2005.

From December 1999 to May 2001, he was a Research Engineer with Datang Technology Company, Ltd., Beijing, China, where he participated in drafting and amending the third-generation time-division synchronous code-division multiple-access (TDSCDMA) standard and in prototyping the TD-SCDMA/GSM system. From January to July 2002, he was a Hardware Research Engineer with Holley Company, Ltd., where he developed the dual-mode handset in cooperation with the Research and Development Department in Dallas, TX. From July 2005 to August 2006, he was a Research Scientist with the Alcatel Shanghai Bell Research and Innovation Center, where he focused on the prototyping of multiple-input-multiple-output orthogonal frequency-division multiplexing (MIMO-OFDM) systems for future fourth-generation systems and worked on the projects of the radio-over-fiber and distributed antenna system. Since September 2006, he has been with Villanova University, Villanova, PA, where he is currently a Postdoctoral Research Fellow with the Center for Advanced Communications. He has published more than 50 papers. His research interests include MIMO, OFDM, radio frequency identification, wireless channel modeling, source localization and tracking, cooperative communications, advanced signal processing and applications, communication network, ad hoc and mesh networks, and circuit and antenna design.

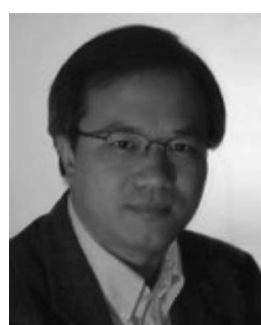

Yimin Zhang (SM'01) received the Ph.D. degree from the University of Tsukuba, Tsukuba, Japan, in 1988

In 1988 , he was with the faculty of the Department of Radio Engineering, Southeast University, Nanjing, China. He served as a Technical Manager with the Communication Laboratory Japan, Kawasaki, Japan, from 1995 to 1997 and was a Visiting Researcher with ATR Adaptive Communications Research Laboratories, Kyoto, Japan, from 1997 to 1998. Since 1998, he has been with Villanova University, Villanova, PA, where he is currently a Research Associate Professor with the Center for Advanced Communications. $\mathrm{He}$ is an Associate Editor for the Journal of the Franklin Institute and serves on the editorial board of the Signal Processing Journal. His research interests are statistical signal and array processing for communications and radar applications, including digital mobile communications, wireless networks, multiple-input-multipleoutput systems, cooperative communications, blind signal processing, jammer suppression, time-frequency analysis, source localization and target tracking, radio frequency identification, and image processing.

Dr. Zhang is an Associate Editor for the IEEE Signal Processing LETTERS.

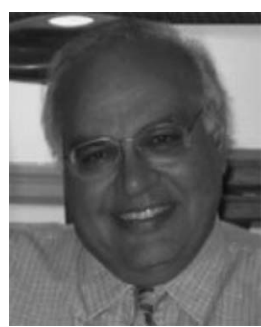

Moeness G. Amin (F'01) received the Ph.D. degree from the University of Colorado, Boulder, in 1984.

Since 1985, he has been with the faculty of Villanova University, Villanova, PA, where is currently a Professor with the Department of Electrical and Computer Engineering and the Director of the Center for Advanced Communications. He has more than 400 publications in the areas of wireless communications, time-frequency analysis, smart antennas, interference cancellation in broadband communication platforms, direction finding, Global Positioning Satellite technologies over the horizon radar, and radar imaging. He was a Guest Editor for the Journal of Franklin Institute September 2008 Special Issue on Advances in Indoor Radar Imaging. He is a Co-Guest Editor for the upcoming IET Signal Processing Special Issue on Time-Frequency Approach to Radar Detection, Imaging, and Classification.

Dr. Amin is a Fellow of the International Society of Optical Engineering. He was a Member of the IEEE Signal Processing Society Technical Committee on Signal Processing for Communications during 1998-2002 and the IEEE Signal Processing Society Technical Committee on Statistical Signal and Array Processing during 1995-1997. He was a Distinguished Lecturer of the IEEE Signal Processing Society for 2003 and 2004 and a Member of the Franklin Institute Committee on Science and the Arts. He is currently a Guest Editor for the IEEE Transactions on Geoscience and Remote SEnsing May 2009 Special Issue on Remote Sensing of Building Interiors and was an Associate Editor for the IEEE TRANSACTIONS ON SIGNAL PROCESSING during 1996-1998. He was a Co-Chair of the Special Sessions of the 2008 IEEE International Conference on Acoustics, Speech, and Signal Processing (Nevada); the Technical Chair of the Second IEEE International Symposium on Signal Processing and Information Technology (Morocco, 2002); the General and Organization Chair of the IEEE Workshop on Statistical Signal and Array Processing (Pennsylvania, 2000); and the General and Organization Chair of the IEEE International Symposium on Time-Frequency and Time-Scale Analysis (Pennsylvania, 1994). He was the recipient of the 2009 Individual Technical Achievement Award from the European Association of Signal Processing, the IEEE Third Millennium Medal, the 1997 Villanova University Outstanding Faculty Research Award, and the 1997 IEEE Philadelphia Section Service Award. 